\title{
El Videoblog en el Aula de Lengua y Literatura de Secundaria. Una Propuesta Práctica
}

\section{Videoblogging in Secondary Education Language and Literature Classroom. A Practical Design}

\author{
Montserrat Blanch Marcos de León * \\ Sonia Betancort Santos \\ Mayra Martínez Avidad \\ Universidad Camilo José Cela
}

\begin{abstract}
Este artículo ofrece estrategias para la alfabetización mediática, informacional y tecnológica a través del uso del videoblog en el aula de Lengua y Literatura de Secundaria. El objetivo es dar, con ello, respuesta a una demanda educativa actual: encontrar fórmulas para fomentar una competencia comunicativa integral en los nuevos entornos multimedia, a la vez que se desarrolla una actitud crítica sobre el consumo de información tradicional y digital. Los primeros resultados obtenidos de la práctica educativa puesta en marcha con alumnos de $3^{\circ}$ de la E.S.O. reflejan en general la adquisición, por parte de los alumnos, de habilidades relacionadas con la competencia comunicativa-digital en el siglo XXI.
\end{abstract}

Descriptores: Educomunicación, Alfabetización mediática, Didáctica de la lengua y la literatura, Videoblog, Educación secundaria, TIC.

This paper offers strategies to enhance media, informational and digital literacy through the use of videoblog in Language and Literature classroom. The aim is to meet a current educative demand: finding formulas to encourage a comprehensive communicational competence for new multimedia environments, at the same time that promotes a critic attitude for traditional and digital information consumption. The tentative results of the educational practice that has been developed among the students of $3^{\text {rd }}$ of E.S.O. show that students acquired skills related to the communicative and digital competence in the 21 st century.

Keywords: Educommunication, Media literacy, Language and literature didactics, Videoblog, Secondary education, ICT.

*Contacto: m.blanch@alumno.ucjc.edu

ISSN: $1696-4713$

www.rinace.net/reice/

revistas.uam.es/reice
Recibido: 29 de julio 2015

$1^{\text {a }}$ Evaluación: 20 de octubre 2015

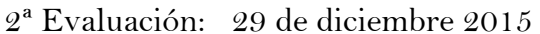

Aceptado: $\quad 4$ de febrero 2016 


\section{Introducción}

La comunicación lingüística destaca dentro del currículo escolar. No sólo como un aprendizaje de la lectura y la escritura, sino como un medio de expresión y empoderamiento que aporta herramientas para comprender la realidad que nos circunda y para seguir aprendiendo durante toda la vida. Daniel Cassany (2009) en una conferencia, lo define así: un "instrumento de estudio y de trabajo para el desarrollo de todos los ámbitos del conocimiento humano, al menos, en nuestra comunidad, en Europa, en Occidente". Sin embargo, no podemos obviar que la Lengua ha encontrado en las TIC nuevos canales y formatos a través de los que representarse, modelos más interactivos de comunicación y de construcción del conocimiento en los que la palabra se entrelaza con el lenguaje audiovisual. Estos nuevos paradigmas exigen un esfuerzo diferente a la hora de leer y comprenderlos, escribir y replicarlos.

En este escenario surge la necesidad de la educomunicación, educación en/o para los medios, campo de estudio surgido en Hispanoamérica en los años setenta y, paralelamente, como media literacy en Estados Unidos. Estos términos tienen sus cimientos en la pedagogía crítica de Paulo Freire (1970) y la pedagogía de la comunicación de Mario Kaplún (1998). Ambos se concretan y unifican en la propuesta de la UNESCO (2011) sobre alfabetización mediática e informacional (AMI) cuya finalidad es "aumentar la conciencia de las múltiples formas que pueden adoptar los mensajes y discursos de los medios de comunicación en el día a día" (Ministerio de Educación, 2011a, p. 33) y capacitar a las personas para ser productoras de información a través de tecnologías y de la Web 2.0.

La escuela de hoy no puede dejar de lado este ámbito de la educomunicación o AMI: es necesario integrar el enfoque tecnológico, ahora preponderante, con otro más crítico, profundo y multimodal. De todo ello depende la verdadera adquisición de la competencia lingüística y comunicativa en la era digital:

La mayor parte de la información que nos llega, así como las comunicaciones, surgen a través de los medios de comunicación y las tecnologías, lo que demanda personas competentes en el uso eficaz y seguro de los medios, haciéndose indispensable una educación, no sólo desde la recepción, desde la decodificación sino a través de la creación, del manejo de los distintos lenguajes y del análisis crítico. (Delgado y Parola, 2013, p. 5)

Por su parte, la asignatura de Lengua y Literatura puede jugar un papel especialmente relevante en la adquisición de esta competencia propia de un contexto y temporalidad profundamente digitales. Más allá de las diferencias en la expresión de los objetivos y contenidos curriculares de esta área para cada una de las Comunidades Autónomas, en todas ellas se destaca el desarrollo de las destrezas comunicativas dentro de un marco que reflexione acerca de los usos lingüísticos sociales como eje vertebrador de la práctica docente. Estos aspectos se reiteran en el Real Decreto por el que se establece el nuevo currículo básico de la Educación Secundaria Obligatoria y del Bachillerato donde se expone que "el objetivo último de esta materia es crear ciudadanos conscientes e interesados en el desarrollo y la mejora de su competencia comunicativa, capaces de interactuar satisfactoriamente en todos los ámbitos que forman y van a formar parte de su vida" (D 1105/2014, p. 191). Asistimos, aunque muy lentamente, a una toma de conciencia sobre la importancia de conjugar el desarrollo de la competencia lingüística con el de la adquisición de destrezas para una comunicación integral. En la línea de lo que señala Prado, y otros autores del ámbito de esta didáctica (Cassany, Sanz y Luna, 
1998; Ruiz Bikandi, 2011) desde enfoques como la lingüística comunicativa, pragmática y funcional:

Ya no es suficiente alfabetizar en el código verbal escrito. Es preciso alfabetizar en otros sistemas verbales, desatendidos tradicionalmente, y no verbales, si no queremos que el alumno se convierta en analfabeto funcional en esta sociedad donde imperan la imagen y el sonido como formas de expresión e información. (Prado, 2004, p. 23)

Se trata de una necesidad cada vez más acuciante si tenemos en cuenta el incremento de información disponible, sobre todo en formato audiovisual, a través de medios digitales y la cantidad de facilidades técnicas, teléfonos móviles inteligentes y tabletas, para acceder a ellos. Por ejemplo, la última medición de la Asociación para la Investigación de los Medios de Comunicación (AIMC, 2015) mostró que Internet, el segundo medio con más consumidores por detrás de la televisión, es uno de los que más crece (un 4\% de usuarios más con respecto al primer resumen del año). Y Youtube fue el sitio de noticias y entretenimiento más visitado con mucha diferencia con respecto a la siguiente web (cuatro veces más visitas que la anterior página listada). Además, muchos periódicos de tradicional tirada en papel están acompañando ya sus artículos en Internet con vídeos, que subrayan y le añaden otra dimensión a la información. Según otro estudio, este de ComScore (2013), los grandes usuarios consumidores de contenidos digitales están en el grupo de entre 15 y 34 años. Sin embargo, pese a la accesibilidad tecnológica y la relativa facilidad para grabar videos y subirlos a la red, el consumo de los jóvenes sigue siendo mayor que la participación y la iniciativa propia subiendo vídeos o escribiendo en blogs (Sánchez Burón y Fernández Martín, 2010). Nos encontramos, por tanto, ante una generación de adolescentes que consume masivamente productos digitales, especialmente vídeos; que tiene unos conocimientos básicos sobre las redes sociales y las funciones que ofrecen las tecnologías móviles; que comparten fotografías y ocasionalmente vídeos; pero que no tienen una conciencia elaborada ni del resultado de sus acciones, ni sobre los contenidos que visionan.

En este sentido, investigaciones recientes (Aguaded et al., 2011; Masanet, Contreras y Ferrés, 2013; Ministerio de Educación, 2011b; Ramírez, Basterretxea y Jiménez, 2011) evidencian que los jóvenes presentan serias dificultades para leer, comprender y evaluar de forma crítica los mensajes de los medios. Un estudio publicado por el Ministerio de Educación en 2011, en el que se evaluó la capacidad crítica de la ciudadanía española frente a los medios, reveló que tan sólo el 6,8\% de los jóvenes de entre 16 y 24 años tenía un nivel equiparable a un suficiente en el conjunto de las capacidades evaluadas. Precisamente esta misma franja de edad alcanzó las puntuaciones más altas con respecto al resto de la población (Ministerio de Educación, 2011b, p. 165). Estas carencias formativas ponen de manifiesto una falta de capacidad crítica y, por tanto, una mayor credulidad ante las imágenes y contenidos presentados. En el marco de estos datos, se ha de tener en cuenta, además, que los textos mediáticos que actualmente se estudian dentro del currículo escolar de Secundaria - la entrevista, la noticia, la crónica, el reportaje- han evolucionado en sus representaciones para los medios digitales, haciéndose más complejos en los formatos y en el lenguaje.

En respuesta a esta situación de analfabetismo mediático, diversas instituciones han lanzado recomendaciones y propuestas en la línea de impulsar la formación de docentes (Ministerio de Educación, 2011 a; Parlamento Europeo, 2008; UnESCO, 2011) para que se puedan incorporar, de forma transversal, contenidos de este ámbito en las diferentes áreas de la enseñanza (LOE, 2006; LOMCE, 2013; Ministerio de Educación, 2011a) e 
incluso, para que la alfabetización mediática sea considerada como una competencia clave para el aprendizaje permanente (Parlamento Europeo, 2008).

Bajo estas perspectivas, presentamos una propuesta didáctica para el aula de Lengua y Literatura de Educación Secundaria: el videoblog como herramienta para promover la alfabetización en los nuevos lenguajes multimedia y el uso crítico de los medios, como parte de los objetivos de aprendizaje que la asignatura debería abordar en el siglo XXI. La experiencia didáctica que sirve para ilustrar estos planteamientos se llevó a cabo con los alumnos de $3^{\circ}$ de la E.S.O del Colegio Julio Verne de Valencia con la colaboración de los profesores del centro educativo y de la Universidad Camilo José Cela.

\section{Fundamentación teórica}

\subsection{Alfabetización mediática y su alcance en la Educación}

Los proyectos de alfabetización mediática han revelado su importancia desde las últimas décadas del siglo pasado. No obstante, con anterioridad, diversos pedagogos sentaron importantes precedentes a tener en cuenta: como Celestin Freinet (1927), con su investigación empírica en torno a la imprenta escolar, o Paulo Freire (1984, 1970), con sus proyectos de alfabetización en adultos y el desarrollo del concepto de la concientización. Ambos ya compartían el mismo objetivo: enseñar a los alumnos a familiarizarse con las formas comunicativas propias de los medios a través de la experiencia en primera persona y la reflexión. Estas prácticas convertían a los estudiantes en los propios productores de los mensajes de los medios, con el fin de que pudieran alcanzar una conciencia más crítica sobre su lenguaje y las relaciones de poder subyacentes.

Desde un punto de vista institucional, a partir de la declaración de Grünwald de 1982 y hasta la declaración de Alejandría de 2005, organismos internacionales y otros actores interesados han venido trabajando en la promoción de estrategias políticas y educativas en torno a la cuestión de la AMI. Así, la UNESCO ha editado una serie de propuestas dirigidas al cuerpo docente con la finalidad de facilitar una integración del currículo en AMI dentro de las materias tradicionales. Sus objetivos se pueden concretar en tres grandes áreas temáticas: "conocimiento y entendimiento de los medios e información para los discursos democráticos y la participación social; la evaluación de los textos mediáticos y fuentes de información; y la producción y uso de los medios y la información" (UNESCO, 2011, p. 23).

En base a esto, distinguimos tres bloques de destrezas específicas que involucran eficazmente a la asignatura de Lengua y Literatura en Educación Secundaria:

- Compresión del contenido de los medios (incluye la capacidad para interpretar los géneros, lenguajes y formatos multimedia de los medios de comunicación).

- Evaluación crítica de la información y de las fuentes de información (incluye la capacidad crítica para discernir la veracidad de la información, los intereses e ideologías ocultas tras las fuentes y la expresión lingüística utilizada). 
- Acceso eficaz a la información y producción de mensajes (incluye la capacidad de uso de las TIC y el correcto empleo de la Lengua).

Precisamente, en la presente propuesta se muestra cómo estas tres dimensiones y sus respectivas destrezas pueden trabajarse de forma integrada y efectiva a través de la asignatura de Lengua y Literatura. Concretamente, se ofrecen una serie de estrategias pedagógicas para dotar a los alumnos de Educación Secundaria de habilidades para la comprensión y evaluación crítica del contenido de los medios, al tiempo que adquieren destrezas para comunicarse en los nuevos formatos y lenguajes digitales, haciendo uso de las herramientas tecnológicas y de una correcta expresión oral y escrita. El proyecto propone así una forma de abordar la competencia lingüística y comunicativa en la era informacional a través de la asignatura de Lengua y Literatura.

\subsection{Una aproximación a la competencia en comunicación lingüística en el siglo XXI}

La metodología empleada en las clases de Lengua ha sido heredera de una tradición academicista y cerrada cuyo énfasis ha consistido principalmente en aprender su estructura: la gramática (Cassany, Sanz y Luna, 1998). Sin embargo, con el discurrir del siglo XX, y principalmente en el último cuarto, la Didáctica de la Lengua y la Literatura ha comenzado a renovarse a través de la incorporación de nuevos supuestos teóricos y metodológicos provenientes de disciplinas como la sociolingüística, la pragmática, la lingüística del texto y la psicolingüística (Martín Vegas, 2009). Así, con respecto al estudio de la estructura de la Lengua, se han adoptado metodologías innovadoras como las surgidas desde los modelos de la gramática comunicacional (Bybee, 2006), el modelo analógico (Skousen, 1995) y la gramática computacional o modelo conectivo (McClelland, Dilkina y Boroditsky, 2007), que destacan la importancia del aprendizaje de las estructuras de la Lengua a través de su uso reflexivo en contextos comunicacionales prácticos, reales y creativos. También a través de propuestas didácticas, como las basadas en la Programación Neurolingüística de Gabarró (2011), que integran la perspectiva psicológica en el estudio de la ortografía.

Del mismo modo, desde el punto de vista de los objetivos, la aparición del enfoque comunicativo ha contribuido a que se preste una mayor atención a la hora de capacitar a los alumnos en el uso práctico de la Lengua:

El enfoque comunicativo en la enseñanza de la Lengua ha puesto de manifiesto que los usos lingüísticos orales y la interacción son la base de la comunicación social y que para alcanzar el pleno desarrollo de la competencia lingüistica de los escolares es necesario mejorar sus destrezas comunicativas, a partir de los conocimientos previos con los que llegan a la escuela. (Prado, 2004, p. 145)

En cuanto a los contenidos, el currículo de Secundaria contempla aspectos relacionados con el estudio formal de los medios. Destaca la aproximación a los géneros periodísticos: la entrevista, la noticia, el reportaje y la crónica, textos que podemos encontrar en los medios de comunicación audiovisual, televisión y radio, y de la prensa escrita. Estos contenidos los podemos encontrar en los Bloques I y II denominados respectivamente "Comunicación oral: escuchar y hablar" y "Comunicación escrita: leer y escribir" (RD $1105 / 2014)$. En este sentido, algunas de las propuestas más atractivas son el desarrollo de talleres de prensa, radio y televisión (Pérez Rodríguez, 2004, 2002). No obstante, el gran reto en el aula reside todavía en formar a los alumnos en las nuevas competencias 
lingüísticas y comunicativas que se requieren hoy día para consumir y producir información en la red.

En los últimos años las TIC también han irrumpido en el aula de Lengua y Literatura. Así lo demuestran las numerosas publicaciones en torno a este tema (Estévez, 2012; Larequi, 2009; Tíscar 2009; Torrego, 2012; Vivancos, 2008) y la consideración de estos recursos en los principales manuales de Didáctica de la materia (Martín Vegas, 2009; Prado, 2004; Ruiz Bikandi, 2011). Pese a esto, y a la riqueza de algunas propuestas, en muy pocas ocasiones se tiene como objetivo definido el desarrollo de destrezas directamente asociadas a la alfabetización mediática (Romero y Doménech, 2010).

En ese marco, la presente propuesta ofrece una experiencia en la que los cibermedios se integran en la propia metodología con el fin de convertirse en objeto mismo de aprendizaje. Para ello, se recurre a una herramienta digital, de gran potencial y riqueza, que reúne todas las características multimedia así como las capacidades de participación y creación necesarias para dicha alfabetización en el aula de Lengua: el eduvlog.

\subsection{El eduvlog, una herramienta innovadora para el aula de Lengua y Literatura}

Los "vlogs", acrónimo formado a partir de la palabra videoblog, son una variante de los populares blogs que tanto éxito han tenido en multitud de proyectos educativos innovadores. En efecto, estos espacios han destacado como instrumentos en diferentes áreas del conocimiento, por tratarse de un recurso accesible, motivador y facilitador de los distintos tipos de alfabetización (Durán, 2010; Santoveña, 2011; Sobrino 2013).

El fenómeno blogging ligado a la educación se produjo primeramente en el ámbito anglosajón. En España, la introducción de los blogs como herramienta educativa llegó más tarde, y al principio los usos se centraron en los niveles superiores de la enseñanza (Tíscar, 2005). Los edublogs - acrónimo formado a partir de las palabras inglesas educational y blog - pueden tener múltiples usos, atendiendo a modelos de comunicación de tendencia unidireccional - tales como publicar información y otros materiales didácticos - hasta los más interactivos: crear grupos de discusión, pedir posibles respuestas o soluciones a un problema planteado, colgar resultados de proyectos para que otros miembros autorizados del blog puedan dejar sus comentarios, etc.

La característica diferenciadora del vlog frente al blog es que, como indica su propio nombre, usa fundamentalmente el vídeo en lugar de la palabra escrita para comunicar. La realización de un eduvlog, con "v", es, de entrada, más compleja que la de un edublog, con "b". En este sentido, además de planificar, acordar temas, buscar y organizar la información, esta se ha de "guionizar" en un texto escrito que ayude al alumno a expresarse oralmente frente a la cámara. Y una vez hecho esto, se ha de grabar, editar, publicar y hacer un seguimiento del impacto que ha generado. Se trata por tanto de actividades en las que el lenguaje textual interactúa con el audiovisual. Durante esta serie de procedimientos de creación informativa y literaria, los estudiantes asimilan las destrezas y los contenidos de la materia de Lengua y Literatura y, acompañados por el profesor, pueden iniciar una reflexión metalingüística sobre las llamadas "subcompetencias morfosintáctica, léxica y ortográfica" (Prado 2004, pp. 255-256). De esta forma se refuerzan la adquisición de conocimientos sobre el uso práctico de la Lengua y el proceso de lectura crítica. Además, el videoblog añade la dimensión audiovisual que enriquece el texto inicial, añadiéndole diferentes matices y perspectivas que habilitan posibilidades de meta-cognición sobre la expresión oral. Por último, a 
través de la publicación de contenidos, los estudiantes pueden acercarse a los procedimientos reales y actuales de producción de los diferentes medios, así como al uso de los géneros periodísticos que estudian.

Así, podemos encontrar que el vlog, como herramienta de apoyo educativo, ha sido utilizada en algunos proyectos relacionados con la didáctica para la adquisición de una segunda Lengua en Secundaria (Garay y Castaño, 2013). También existe evidencia de su uso en niveles superiores de la enseñanza con resultados muy satisfactorios en cuanto a su efectividad como elemento motivador y coadyuvante del aprendizaje (García, Melendo, Presol, 2013). Otra autora, Meeder, ha estudiado la aplicación de la herramienta en instituciones de Educación Secundaria y Universidades de Estados Unidos y Japón. En su investigación destaca el gran potencial de este recurso didáctico que enriquece la experiencia educativa para profesores y alumnos, e incluso para las familias ya que añade lo atractivo del elemento visual en la representación de resultados y aprendizajes (2008, p. 17). Las conclusiones de Meeder son confirmadas por García, Melendo y Presol (2013, p. 166), que señalan entre las ventajas de esta herramienta un mayor dinamismo y motivación en el trabajo con los alumnos, la mejora de la competencias comunicativas y de la alfabetización tanto informacional como digital, así como interesantes posibilidades para diversificar las formas de evaluación a través de los comentarios en línea, bien por parte del docente o de los compañeros.

\section{Una experiencia de aplicación del videoblog en el estudio de los géneros periodísticos en $3^{\circ}$ de la ESO}

\subsection{Contexto}

La práctica está dirigida a los alumnos de $3^{\circ}$ de la E.S.O del Colegio Julio Verne (Valencia), Centro de titularidad privada. Los elementos característicos del ideario del colegio son la inclusión, la promoción de valores democráticos y el desarrollo de proyectos innovadores que formen integralmente al alumnado. Asimismo, el Centro apuesta por la aplicación de metodologías y pedagogías renovadoras adaptadas a las necesidades de los alumnos. Reflejo de ello es que las clases se apoyan comúnmente en metodologías de aprendizaje cooperativo y M.O.R.E (Múltiples Opciones para mejorar los Resultados Educativos), buscando formar individuos libres y responsables.

\subsection{Objetivos y competencias}

Organizamos los objetivos en base a los tres bloques de destrezas específicas identificadas más arriba como parte de la competencia lingüística en la era informacional (tabla 1).

\subsection{Contenidos}

Los contenidos conceptuales sobre los que se desarrolla esta práctica son los géneros periodísticos, más específicamente la entrevista, la noticia y el reportaje. A partir del análisis de estos textos, escritos y audiovisuales, se inicia una reflexión metalingüística a través de la cual se refuerzan y corrigen errores en los conocimientos gramaticales previos de los alumnos acerca del uso de los tiempos verbales, los tipos de oración (activas y pasivas), los tipos de palabras, la ortografía y los signos de puntuación. 
Los contenidos procedimentales se centran en el desarrollo de las capacidades expresivas de los alumnos. Tanto en un medio escrito: redacción de baterías de preguntas para preparar una entrevista, noticias, reportajes y resúmenes de textos periodísticos; como en el discurso oral (“oralización”) de los textos previamente preparados: discusión y debate sobre conceptos gramaticales, definiciones sobre géneros periodísticos y sus funciones etc. Así mismo se ejercitan las habilidades de leer y comprender: búsqueda, filtraje y lectura de textos periodísticos; y de escuchar: ejemplos de cada género, escucha activa en el aula etc. Para todo ello se proponen diversas actividades encaminadas a la elaboración de videoblogs en los que los alumnos deben recrear los textos periodísticos que se estudian, darles un formato audiovisual y compartirlos en las redes sociales.

En cuanto a los contenidos actitudinales, la propuesta refuerza una constante reflexión, y fomenta la actitud comprometida y responsable con respecto al trabajo individual y en grupo. De este modo, se promueve la actitud crítica, constructiva e indagadora, que permite al alumnado adquirir recursos de meta-cognición.

Tabla 1. Objetivos de la propuesta

\section{COMPRENSIÓN DEL CONTENIDO DE LOS MEDIOS}

- Leer y comprender textos procedentes de los medios de comunicación audiovisual.

- Identificar correctamente los géneros periodísticos y los subtipos que se van a estudiar: la noticia, la entrevista y el reportaje.

- Reflexionar sobre el uso del lenguaje: la gramática (verbos, pronombres, adjetivos y adverbios) y el uso de la puntuación y la ortografía en los medios de comunicación audiovisual y en Internet.

- Descifrar contenidos en formatos multimedia (música, imágenes, efectos, vídeos, gráficos interactivos etc.).

EVALUACIÓN CRÍTICA DE LA INFORMACIÓN Y DE LAS FUENTES

- Contrastar informaciones, buscar fuentes originales, discernir la fiabilidad de la información.

- Reflexionar sobre la naturaleza de las informaciones en los medios.

- Desarrollar una visión crítica sobre los contenidos presentados por los medios.

- Aplicar una mirada crítica sobre la expresión lingüística utilizada en los medios. ACCESO EFICAZ A LA INFORMACIÓN Y PRODUCCIÓN DE MENSAJES

- Acceder a noticias, acontecimientos e información de interés.

- Utilizar la tecnología apropiada para componer textos propios de los medios de comunicación audiovisual.

- Saber producir y difundir contenidos digitales y audiovisuales a través de los cibermedios de comunicación social.

- Componer y corregir textos propios de los medios de comunicación (entrevistas, noticias y reportajes) aplicando las normas gramaticales, y los usos de ortografía y puntuación oportunos.

Fuente: Elaboración propia.

\subsection{Fases y metodología}

La práctica se divide en tres etapas: fase inicial o de observación del contexto educativo sobre el que se diseña la propuesta, fase intermedia o de desarrollo de la actividad y fase final o de evaluación.

\subsubsection{Fase inicial}

Con el fin de detectar los hábitos de consumo de medios de los adolescentes, así como sus conocimientos tecnológicos, se propuso llevar a cabo un cuestionario previo. Los aspectos sobre los que se preguntó fueron los conocimientos tecnológicos, el consumo de 
medios, las preferencias culturales así como la percepción de la materia de Lengua y Literatura.

Asimismo, en esta primera fase se analizaron las programaciones de la asignatura de Lengua y Literatura y se observó el estilo pedagógico del profesorado en torno a las tres líneas principales que identificamos como ejes de la competencia lingüísticainformacional:

- Comprensión del contenido de los medios: La comprensión lectora de textos en esta asignatura se daba, sobre todo, a partir de formatos impresos. Los textos mediáticos se estudiaban únicamente dentro de uno de los bloques de contenido, el establecido en el currículo. Por otra parte, el análisis de la gramática y de la ortografía estaba vinculado a modelos didácticos tradicionales en los que se memorizan las funciones de los diferentes elementos y su uso correcto. Con todo, se observó el empleo de recursos lúdicos como juegos y actividades interactivas que rebasaban el enfoque tradicional.

- Evaluación crítica de la información y de las fuentes: No se observó un trabajo concreto para esta área. Primaba una enseñanza erudita, de comprensión y memorización de contenidos fijos. Asimismo, se observó el esfuerzo del profesorado por ofrecer esquemas y resúmenes para aclarar y ayudar a la memorización de los contenidos estables. Estas actitudes, aunque positivas para otros objetivos, no fomentan el desarrollo de habilidades para discernir la veracidad de las fuentes.

- Acceso eficaz a la información y producción de mensajes: Se apreció una voluntad de dar libertad al alumnado a la hora de buscar información, sintetizarla y preparar presentaciones. Estas se daban mayormente en formatos no digitales, preferentemente presentaciones en Power Point y en la plataforma Prezi. Se dedicaba más tiempo y recursos a la práctica de la habilidad lectora que a la oral.

A partir los resultados del cuestionario y de estas observaciones se propuso la práctica pedagógica para el estudio de los géneros periodísticos a través del uso del videoblog.

\subsubsection{Fase de desarrollo}

Dado que la elaboración de un video es una práctica compleja, se propuso que los alumnos trabajasen en grupos (de cuatro, y excepcionalmente de tres), repartiéndose las tareas. Se dio libertad al alumnado para elegir los temas sobre los que investigar y redactar sus trabajos. Por su parte, los profesores asumieron un rol de orientadores, incitadores a la reflexión sobre las tareas y mediadores ante los conflictos internos de los grupos.

Como se puede observar en la tabla 2, la propuesta consistió en seis sesiones de una hora, una vez a la semana, en semanas correlativas: cuatro sesiones principales, una de apoyo intercalada, y una para que los alumnos evaluasen sus proyectos. Las sesiones se basaron en puestas en común acerca de los diferentes géneros periodísticos, en las que se dieron a conocer instrumentos y estrategias audiovisuales y digitales para su comprensión y posterior elaboración. Para el análisis de los contenidos audiovisuales en entrevistas, noticias y reportajes, se seleccionaron fragmentos procedentes 
principalmente de routube.com. Se analizaron elementos y funciones textuales, macroestructura y microestructura de los textos, expresión oral y lenguaje no verbal.

Durante la primera sesión, los alumnos crearon sus plataformas de trabajo (blogspot.com, blogger.com o canal en Toutube). Se enfatizó el trabajo colaborativo y la toma de decisiones sobre las plataformas digitales para compartir contenidos y el diseño de las mismas. Durante la segunda sesión, se analizó, en grupo, el género de la entrevista, las características y funciones de esta, y se programó la realización de la primera práctica: una encuesta o indagación a un especialista de algún campo. Para la presentación de la actividad se debía justificar la elección del tema y del entrevistado, la recogida y análisis de información, y las conclusiones del proceso.

Tabla 2. Cronograma general de actividades

\begin{tabular}{|c|c|}
\hline SESIONES & ACTIVIDADES \\
\hline $1^{\text {a }}$ sesión & $\begin{array}{l}\text { Presentación del proyecto. } \\
\text { Práctica grupal: creación y diseño de plataformas de trabajo (blogspot.com, } \\
\text { blogger.com o canal en } \text { Toutube). }\end{array}$ \\
\hline $2^{\mathrm{a}}$ sesión & $\begin{array}{l}\text { Estudio del género entrevista: tipos, características, estructura y funciones. } \\
\text { Práctica grupal (se completa fuera del aula): grabar y editar un cuestionario o } \\
\text { una entrevista a experto y subirlo a la plataforma. }\end{array}$ \\
\hline $3^{\mathrm{a}}$ sesión & $\begin{array}{l}\text { Estudio del género noticia: características, estructura y funciones. } \\
\text { Práctica grupal (se completa fuera del aula): seleccionar una noticia de los } \\
\text { medios impresos, reconstruirla para grabarla y editarla en vídeo, y subirla a la } \\
\text { plataforma }\end{array}$ \\
\hline $4^{\mathrm{a}}$ sesión & Revisión del trabajo realizado, valoración y resolución de las dificultades. \\
\hline $5^{\text {a }}$ sesión & $\begin{array}{l}\text { Estudio del género reportaje: tipos, características, estructura y funciones. } \\
\text { Revisión y comparación de los tres géneros periodísticos. } \\
\text { Práctica grupal: planteamiento y redacción de un principio de reportaje para } \\
\text { televisión. }\end{array}$ \\
\hline $6^{\text {a }}$ sesión & Autoevaluación y coevaluación de las plataformas y proyectos realizados. \\
\hline
\end{tabular}

Fuente: Elaboración propia.

La tercera y cuarta sesión se orientaron hacia el estudio de la noticia, su estructura externa e interna, los tipos de oraciones, las formas verbales y las diferencias entre el texto oral y escrito. A partir de esta puesta en común, cada grupo elaboró su segunda práctica: una noticia grabada. La cuarta sesión se dedicó al asentamiento de los contenidos vistos y a la resolución de dificultades conceptuales, técnicas y de funcionamiento de los grupos.

Durante la quinta sesión, se abordó el estudio del reportaje, un género periodístico complejo por la cantidad de elementos y lenguajes que aglutina. Textos procedentes de los medios escritos y audiovisuales, reportajes y crónicas digitales, sirvieron para recorrer las características más relevantes de esta modalidad. Se instó a los alumnos a relacionar los conceptos aprendidos hasta la fecha, las diferencias y coincidencias de los géneros estudiados y los formatos elegidos en puestas en común.

Finalmente, la sexta sesión se dedicó a la autoevaluación y coevaluación de los proyectos: primeramente los grupos expusieron sus trabajos frente al resto de la clase, reflexionaron y realizaron evaluaciones sobre sus prácticas. Después de esta valoración, los demás compañeros aportaron otras críticas, siempre razonadas, o soluciones a problemas que había afrontado el grupo sin éxito. A parte de la expresión oral, también se valoraron elementos no verbales, paraverbales y técnicos. Los criterios de evaluación 
empleados fueron las directrices que previamente se habían dado a los alumnos para realizar cada una de las prácticas.

Además de estas actividades en grupo, se pidió a los estudiantes que realizaran, de forma voluntaria, un vídeo individual con una duración de entre uno y tres minutos, y una estructura narrativa básica (introducción, desarrollo y conclusiones). A través de este mecanismo se esperaba que ofrecieran una breve reflexión personal evaluando la marcha del proyecto y una valoración sobre su propio aprendizaje.

\section{Materiales y recursos}

Para el desarrollo de la propuesta se ha contado con el equipamiento técnico de aula (pizarra, pizarra digital, ordenadores de aula, Internet y libros de texto), así como los recursos propios de los alumnos (móvil, Internet, ordenadores de casa, memorias portátiles digitales, cámaras de vídeo, webcams y cámaras fotográficas).

Asimismo se precisaron diversos recursos bibliográficos y audiovisuales: sitios web de medios de noticias nacionales, locales y de prensa especializada (elpais.com, elmundo.es, lasprovincias.es, levante-emv.com, marca.com etc.) y de noticias de entretenimiento (elmundotoday.com), textos audiovisuales procedentes de diferentes medios digitales como los repositorios de RTVE, Antena3, LaSexta o vídeos de Youtube.com; el sitio web del diccionario de la RAE (http://www.rae.es/) y actividades de la web del Instituto Cervantes sobre la puntuación en los medios de comunicación (http://cvc.cervantes.es/actcult/morderse_lengua/).

\subsubsection{Metodología}

Como puede observarse, la estrategia metodológica empleada se acerca a los principios del enfoque constructivista: se ha perseguido un aprendizaje significativo, partiendo de conocimientos e intereses previos del alumnado que, con el apoyo del profesororientador como facilitador del proceso, ha elaborado sus propios contenidos. Por otra parte, con respecto a la materia de Lengua y Literatura, predomina un enfoque comunicativo, la reflexión meta-lingüística y una metodología deductiva. Se ha puesto el acento en la toma de consciencia sobre el acto comunicativo. Con el apoyo de contextos reales y de la materialización de micro y macro tareas se han reelaborado los conceptos de la Unidad. Este tipo de procesos implica ser flexibles con los espacios y tiempos de trabajo, por este motivo y acorde con la metodología expuesta, dichas variables han sido distribuidas de manera autónoma por los alumnos: las grabaciones, la edición y la subida de contenidos se llevan a cabo fuera de los horarios de clase; y los escenarios implican actividad dentro y fuera del aula (el resto del centro escolar, la casa, la calle, Internet).

\subsection{Fase de evaluación}

Para la evaluación se propusieron diferentes instrumentos de tipo cualitativo y cuantitativo:

- Cuestionario final a alumnos y profesor en el que se miden las valoraciones personales en cuanto a los puntos fuertes y débiles del proyecto, así como la percepción sobre la efectividad del proyecto y de la herramienta sobre sus propios aprendizajes.

- Observación participante y entrevistas informales con el profesor de Lengua y literatura, con otros profesores, y con los propios alumnos. A 
través de una hoja de registro se toma nota de la actitud, participación e implicación individual y grupal durante las clases, el interés, la proactividad, la iniciativa, el dinamismo, la resolución de problemas técnicos y conflictos personales, el respeto, el compromiso y el comportamiento crítico-reflexivo. Se elaboró una rúbrica para evaluar el comportamiento de cada alumno y el funcionamiento de los grupos.

- Estimación de la consecución de los objetivos de aprendizaje a través de las propias prácticas realizadas por los alumnos durante cada una de las sesiones de trabajo, así como de pruebas objetivas sobre los contenidos impartidos. Para valorar las prácticas se realizó una rúbrica que valoraba el nivel de la planificación de los trabajos y su resultado final reflejado en la expresión oral, elementos no verbales, paraverbales y técnicos.

\section{Resultados}

La propuesta se llevó a cabo con treinta y cuatro alumnos (34) divididos en dos grupos de dieciséis (16A) y dieciocho (18B), de edades entre los 14 y 15 años. La intervención se realizó en sesiones de una hora a la semana en el horario de la asignatura de Lengua y Literatura durante un período de tres meses.

Los resultados de la evaluación inicial sobre los hábitos de uso y consumo de medios de los alumnos arrojaron los siguientes datos: existía entre los alumnos un amplio acceso a la tecnología y al consumo de contenidos audiovisuales desde el ordenador personal, el teléfono inteligente gama media-alta, la conexión a Internet y la televisión. Estos alumnos utilizan principalmente el teléfono móvil, la cámara de fotos $(97 \%)$ y la cámara de video (94\%), y en menor medida, llevan a cabo la edición y manipulación de archivos $(69 \%)$. En cuanto a la edición por ordenador, los estudiantes manipulan eventualmente fotografías usando Paint y Photoshop (71\%) y con menor regularidad video con Windows Video Maker (26\%). Las tres redes sociales más utilizadas por estos jóvenes son Twitter, Instagram y Youtube. Con respecto a Youtube, y a diferencia de las otras dos redes, el uso de la plataforma confirma a estos estudiantes más como consumidores de vídeo que como productores de contenido. En cuanto a la valoración de la asignatura de Lengua y Literatura, el informe revela una preferencia de las clases participativas y prácticas (exposiciones, lectura y estudio de la ortografía) frente a otras de índole más teórica (estudio de la sintaxis y de la literatura).

Tras la intervención, el logro de los objetivos pedagógicos relativos a la competencia lingüística en la era informacional se evaluó a través de las prácticas y pruebas objetivas, tal y como se observa en la tabla 3.

Los resultados de este proyecto piloto permiten asumir que la propuesta impacta en la dirección deseada. Las puntuaciones obtenidas en las prácticas, así como los resultados del examen trimestral reflejan en general la adquisición, por parte de los alumnos, de habilidades relacionadas con la competencia comunicativa-digital. 
Tabla 3. Aspectos evaluados en las prácticas y en la prueba objetiva

\begin{tabular}{ll}
\hline \multicolumn{1}{c}{ PRÁCTICA } & ASPECTOS EVALUADOS \\
\hline Videoblog & $\begin{array}{l}\text { Diseño (fondos, fuentes, imágenes, gráficos, etc.) } \\
\text { Funcionalidad (facilidades para interactuar con otros usuarios) }\end{array}$ \\
\hline & $\begin{array}{l}\text { Estructura del texto (introducción, apartados y reflexión final) } \\
\text { Contenidos lingüísticos (correcta expresión oral y escrita, coherencia } \\
\text { narrativa, adecuación del texto, corrección gramatical y ritmo) } \\
\text { Antrevista, noticia, } \\
\text { reportaje }\end{array}$ \\
$\begin{array}{l}\text { Aspectos técnicos (coherencia y adecuación de las imágenes con } \\
\text { respecto al texto, calidad audiovisual). }\end{array}$ \\
$\begin{array}{l}\text { Selección adecuada de las fuentes informativas y su citación. } \\
\text { contenidos de la } \\
\begin{array}{l}\text { Unidad en el examen } \\
\text { final trimestral }\end{array}\end{array}$ & $\begin{array}{c}\text { Identificación de un texto periodístico y sus características esenciales: } \\
\text { corrección en la identificación del género, razonamiento adecuado, }\end{array}$ \\
\hline
\end{tabular}

Fuente: Elaboración propia.

Con respecto a los objetivos pedagógicos marcados para la "Comprensión del contenido de los medios":

- El 100\% de los alumnos identifica correctamente el tipo y subtipo del texto periodístico. Y de ese porcentaje, solo un 23\% responde de manera incompleta la pregunta de la Unidad sobre las características de dicho tipo de texto. Durante el proceso, los estudiantes han protagonizado acciones metalingüísticas observando la expresión de los medios de comunicación audiovisual y en Internet.

En cuanto a los objetivos pedagógicos marcados sobre la "Evaluación crítica de la información y de las fuentes de la información":

- El $88 \%$ de los alumnos reflexionó sobre la función de los textos periodísticos, su calidad lingüística y de contenido, y la cantidad de mensajes que o bien consumimos, o que producimos y que también compartimos durante actividades de análisis y reflexión diseñadas para tal efecto realizadas en el aula.

- $\mathrm{El} 66 \%$ de los alumnos investigó sobre informaciones de su interés e hizo un contraste mínimo de fuentes de las que había obtenido la información y sobre su fiabilidad.

Por último, con respecto al "Acceso eficaz a la información y producción de mensajes":

- Un 91\% de los alumnos se familiarizó con tareas tales como abrir un espacio propio para publicar contenidos, grabar y montar videos.

- La media en la entrega y corrección lingüística y de contenido de las prácticas fue del $77 \%$ (92\% en el caso de la entrevista, $41 \%$ en el caso de la noticia y $97 \%$ en el caso del reportaje).

En cuanto a la valoración del profesorado sobre el impacto y la marcha general del proyecto, aprecia una conexión entre los saberes académicos y la práctica de la Lengua en contextos reales y observa mejoría en la expresión oral del alumnado, para actividades que conlleven ciertas pautas (preparación de borradores de ideas previos, trucos para hablar en público y estar relajado, cuidado en la dicción etc.). 


\section{Conclusiones}

Todo parece indicar que el mejor camino para que los alumnos adquieran y desarrollen la competencia mediática es la práctica. A través del uso del videoblog se puede facilitar la reflexión y la activación de procesos de meta-cognición a partir de la escucha y visionado de la propia expresión oral. Esto es, que este tipo de acciones permite a los alumnos tomar consciencia sobre su expresión lingüística y facilita la detección de errores comunes, un interesante punto de partida para la mejora de su destreza comunicativa. Asimismo, tanto la investigación como el soporte digital utilizado, o las prácticas de expresión oral y escrita sujetas al mismo, han supuesto un excelente instrumento de reflexión sobre el uso de la Lengua. En un contexto y tiempo reales, cercano a los alumnos, lejos de las abstracciones de la enseñanza tradicional de la gramática, se ha visto que el análisis y la práctica de la sintaxis y la puntuación revela un alto rendimiento del vlog, un activo facilitador del aprendizaje desde un enfoque comunicativo, funcional y pragmático de la Lengua.

Por otra parte, se ha evidenciado la importancia de la planificación y análisis del discurso. El uso de esta herramienta aporta un conocimiento en primera persona que implica tanto el proceso de producción de textos, en este caso la entrevista, la noticia y el reportaje, como el de la edición. Con esto se logra que los alumnos aperciban cómo los hechos, a través de distintos lenguajes, pueden manipularse en determinadas ocasiones. De este modo, este tipo de propuestas impulsan una reflexión constructiva que eleva el nivel de consciencia de los estudiantes, concientizar que diría Paulo Freire, al tiempo que aprenden estrategias para mejorar su expresión y su conocimiento de la Lengua. Todo esto nos conduciría al empoderamiento de los jóvenes, futuros ciudadanos de pleno derecho, ya que sin una adecuada formación desde esta perspectiva, pueden quedar desvalidos en un contexto cada vez más tecnológico, competitivo y cambiante.

A pesar de las limitaciones de tiempo en las que se desarrolló la propuesta presentada, podemos confirmar las ventajas del vlog señaladas en el marco teórico por García, Melendo y Presol (2013). Destacamos, por otra parte, que es una necesidad ineludible la formación de profesorado, en activo y en las universidades para que se puedan implementar proyectos de este tipo y para que se pueda orientar adecuadamente al alumnado. Asimismo, estas actividades, dada la gran cantidad de áreas de conocimiento que activan, pueden ser una oportunidad para que docentes de diversas materias colaboren entre ellos.

Por todo ello, esta propuesta constituye una invitación a nuevas experiencias del uso de herramientas digitales e interactivas en la etapa de Educación Secundaria, pues los resultados confirman el gran potencial del videoblog como un instrumento que tiene mucho que ofrecer, y que puede ser útil no sólo para la Didáctica de la Lengua y la Literatura, sino también para otras Didácticas. 


\section{Referencias}

Aguaded Gómez, J. I., Ferrés y Prats, J., Cruz Díaz, M. R., Pérez Rodríguez, M. A., Sánchez Carrero, J. y Delgado, A. (2011) El nivel de competencia mediática en la ciudadanía andaluza. Sevilla: Grupo Comunicar Ediciones.

AIMC (2015). Resumen general de resultados EGM. Febrero a Noviembre 2015. Recuperado de http://www.aimc.es/

Bybee, J. (2006). From usage to grammar: the mind's response to repetition. Language, 82(4), 711-733. doi:10.1353/lan.2006.0186

Cassany, D., Sanz, G. y Luna, M. (1998). Enseñar lengua. Barcelona: Graó.

ComScore. (2013). Spain digital future in focus 2013. Recuperado de http://www.comscore.com/esl/

Delgado, A. y Parola, A. (2013). La urgencia de la competencia mediática en los programas escolares. Revista Chasqui, 124, 4-10. doi:10.16921/chasqui.voi124.12

Durán, J. F. (2010). La utilización de los edublog en las aulas como dinamizador del proceso de enseñanza-aprendizaje. Revista Docencia e Investigación, 20, 205-243.

Estévez, M. E. (2012). Análisis y beneficios de la incorporación de las TIC en el área de Lengua Castellana y Literatura: un caso práctico. Pixel-Bit: Revista de medios y educación, 40, 21-34.

Freire, P. (1970). Pedagogía del oprimido. Recuperado de http://www.omegalfa.es

Freire, P. (1984). La importancia de leer y el proceso de liberación. Madrid: Editorial Siglo Veintiuno.

Freinet, C. (1927). L'imprimerie à l'école. Boulogne: Ferrary éditeur.

Gabarró, D. (2011). Recursos educativos prácticos con programación neurolingüística. Recuperado de http://www.edudigital.es/es/gabarro/

Garay, U. y Castaño, C. (2013). Uso del videoblog para un aprendizaje colaborativo de segundas lenguas con alumnado inmigrante. EDUTEC. Revista Electrónica de Tecnología Educativa, 46 , art 5 .

García, E., Melendo, L. y Presol, A. (2013). Recursos audiovisuales en la docencia a nivel universitaria. El uso del videoblog como herramienta de comunicación. Historia y Comunicación Social, 18, 159-172. doi:10.5209/rev_hics.2013.v18.44322

Kaplún, M. (1998). Una pedagogía de la comunicación. Madrid: Ediciones de la Torre.

Larequi, E. (2009). Propuestas para la integración curricular de las TIC en el área de lengua castellana y literatura. En F. Zayas, L. Tíscar, N. Alonso y E. Larequi (Comps.), La competencia digital en el área de Lengua (pp. 56-89). Madrid: Octaedro

Martín Vegas, R. A. (2009). Manual de didáctica de la lengua y la literatura. Madrid: Síntesis.

Masanet, M. J., Contreras, P. y Ferrés, J. (2013). Highly qualified students? Research into the media competence level of Spanish youth. Comunicación y Sociedad, 26(4), 217-234. doi:10.1080/14733285.2013.850849

McClelland, J., Dilkina, K. y Boroditsky, L, (2007). How language affects thought in a connectionist model. Recuperado de http://psych.stanford.edu/

Ministerio de Educación. (2011a). Alfabetización mediática y competencias básicas. Proyecto Mediascopio. Recuperado de http://www.campus-oei.org/

Ministerio de Educación. (2011b). Competencia mediática. Investigación sobre el grado de competencia en la ciudadanía en España. Recuperado de http://ntic.educacion.es/ 
Parlamento Europeo. (2008) Resolución sobre la alfabetización de los medios de comunicación en un mundo digital. Recuperado de http://www.europarl.europa.eu/

Pérez Rodríguez, M. A. (2002). La enseñanza de la Lengua y la Literatura con los medios de comunicación para el desarrollo de la competencia comunicativa. Tesis doctoral. Universidad de Huelva, España.

Pérez Rodríguez, M. A. (2004). Los nuevos lenguajes de la comunicación. Enseñar y aprender con los medios. Barcelona: Paidós.

Prado, J. (2004). Didáctica de la Lengua y la Literatura para educar en el s.XXI. Madrid: La Muralla.

Ramírez, T., Basterretxea, J. I. y Jímenez, E. (2011). El estado de la alfabetización audiovisual en la comunidad escolar vasca. Revista Comunicar, XVIII(36), 157-164. doi:10.3916/c36-201103-07

Romero, A. I. y Doménech, L. (2010). Tic y lengua, el reto del cambio metodológico. Cuadernos de Pedagogía, 401, 51-53

Ruiz Bikandi, U. (Coord.). (2011). Didáctica de la lengua castellana y la literatura. Barcelona: Graó.

Sánchez Burón, A. y Fernández Martín, M. (2010). Informe generación 2.O: hábitos de los adolescentes en el uso de las redes sociales. Estudio comparativo entre comunidades autónomas. Recuperado de http://www.eduteka.org/gestorp/

Santoveña, S. (2011). Incidencia de los nuevos alfabetismos en la mejora de la calidad de la enseñanza: el caso de los blogs. Aula Abierta, 39(2), 59-68.

Skousen, R. (1995). Analogy: a non-rule alternative to neural networks. Rivista di lingüistica, 7, 213-231

Sobrino, D. (2013). El trabajo con blogs en ciencias sociales, geografía e historia. Proyecto Clío, History and History Teaching, 39, 57-76.

Tíscar, L. (2005). Blogs para educar. Uso de los blogs en una pedagogía constructivista. Revista Telos, 65, 86-93.

Tíscar, L. (2009). Alfabetizar en la cultura digital. En F. Zayas, L. Tíscar, N. Alonso y E. Larequi (Eds.), La competencia digital en el área de Lengua (pp. 134-154). Madrid: Octaedro.

Torrego, A. (2012). La utilización de los blogs como recurso educativo en el área de Lengua Castellana y Literatura. Revista Electrónica Interuniversitaria de Formación del Profesorado, 15(4),127-137.

UNESCO. (2011). Alfabetización mediática e informacional. Currículum para profesores. París: UNESCO.

Vivancos, J. (2008). Tratamiento de la información y competencia digital. Madrid: Alianza.

\section{Breve CV de las autoras}

\section{Montserrat Blanch Marcos de León}

Doctoranda en Educación, investiga las relaciones entre el cine y la literatura y sus aplicaciones didácticas en Secundaria. Investigadora en formación vinculada a los Grupos de Investigación Cultura, Sociedad y Educación, y Espacios Educativos de Literaturas Interactivas de la Universidad Camilo José Cela (UCJC), ambos proyectos financiados por el Vicerrectorado de Investigación e Innovación de la UCJC. Licenciada en Comunicación Audiovisual por la Universidad de Valencia, también ha cursado 
estudios de Educación Social por la UNED. Ha diseñado y coordinado diversas iniciativas para la alfabetización mediática y audiovisual, dirigidos a menores, jóvenes y adultos, tales como radios escolares, talleres de cortometrajes y animación, y fotografía. Email:m.blanch@alumno.ucjc.edu

\section{Sonia Betancort Santos}

Profesora de Literatura y Didáctica de la Lengua y la Literatura en la Universidad Camilo José Cela (Madrid). Directora del Proyecto de Investigación competitiva "Espacio Educativo de Literaturas Interactivas" (EELI) subvencionado por el Vicerrectorado de Investigación e Innovación y coordinadora de la Sede Ferraz de la Facultad de Educación de dicha Universidad. Doctora en Literatura por la Universidad de Salamanca, centra sus investigaciones en la narrativa y la poesía hispanoamericanas contemporáneas y en el concepto de interactividad aplicado a las letras hispanas y a su didáctica. Ha publicado más de una veintena de artículos, libros y separatas en el campo de la crítica y la creación literaria. Ha realizado numerosas estancias y ha participado en diversos proyectos de investigación en América Latina. Entre el año 2002 y el 2009 coordinó la Sede de la Universidad de Salamanca en Buenos Aires. Email: sbetancort@ucjc.edu

\section{Mayra Martínez Avidad}

Profesora de Sociología, Tecnología y Medios de Comunicación en la Facultad de Ciencias Sociales y de la Educación de la Universidad Camilo José Cela (Madrid). Visiting Student (Becaria de postgrado Fundación Caja Madrid) en el Departamento de Comunicación de la University of California, San Diego, entre el año 2005 y 2007. Ha escrito artículos y participado en congresos internacionales sobre comunicación participativa, redes alternativas de comunicación y poder. Doctora en Sociología de la Comunicación, investiga nuevas formas de comunicación en el ciberespacio, así como su influencia en la constitución de realidad educativa, social y política. Miembro del grupo de investigación "Ciberdemocracia” de la Universidad Rey Juan Carlos, financiado con un proyecto I+D del Ministerio de Economía y Competitividad (2013-2016) y participante en el proyecto de investigación "Espacio Educativo de Literaturas Interactivas" (EELI), financiado por la UCJC. Ha desempeñado labores de comunicación en la Sede de Comisión Europea y ocupado cargos de redacción y edición en varios medios especializados. Email:mmartinez@ucjc.edu 